\title{
Zero Strength of the Lorentz Force and Lack of a Classical Energy Radiation in the Bohr's Hydrogen Atom
}

\author{
Stanislaw Olszewski \\ Institute of Physical Chemistry, Polish Academy of Sciences, Warsaw, Poland \\ Email: olsz@ichf.edu.pl
}

How to cite this paper: Olszewski, S. (2019) Zero Strength of the Lorentz Force and Lack of a Classical Energy Radiation in the Bohr's Hydrogen Atom. Journal of Modern Physics, 10, 717-724.

https://doi.org/10.4236/jmp.2019.107051

Received: May 21, 2019

Accepted: June 16, 2019

Published: June 19, 2019

Copyright (C) 2019 by author(s) and Scientific Research Publishing Inc.

This work is licensed under the Creative Commons Attribution International License (CC BY 4.0).

http://creativecommons.org/licenses/by/4.0/

\begin{abstract}
It is demonstrated that the Lorentz force acting on the electron particle in the Bohr's hydrogen atom, as well as the classical radiation energy of that atom, tends to be zero on condition both the electric and magnetic fields in the atom are considered in a definite quantum state. The ratio of the mentioned fields becomes of importance for discussion of the occurence of the electron spin.
\end{abstract}

\section{Keywords}

Bohr's Hydrogen Atom, Lorentz Force, Classical Electron Radiation Energy, Electron Spin

\section{(c) (i) Open Access}

\section{Introduction}

The Bohr model of the hydrogen atom can be still a useful tool in considering the electron properties in that atom. The point - which was neglected by Bohr and was planned to be discussed in the present paper - concerns mainly the influence of the presence of the magnetic field in the atom on the physical processes which can be examined.

In general the Bohr model is based on the equivalence of the attractive electrostatic force between the electron and atomic nucleus and the repulsive centrifugal force due to the velocity of the circulating electron along its orbit [1-5]. This assumption is supplemented by the quantization requirement concerning the electron angular momentum. In effect it is obtained the well-known set of the electron states of a definite energy, the differences of which remain in a perfect agreement with experiment.

This semiclassical model was formally objected mainly because of the fact that the motion of the electron particle should be accompanied by a continuous emission of its energy which makes the particle motion to be only a very temporary effect. In practice, however, the electron motion in the electron's ground state is never stopped, and in case of an 
excited atom we observe only a very short-time spontaneous transition of the electron from a higher to a lower quantum energy level. The emitted energy in course of such transition is strictly connected with the energy difference existent between the levels.

Our idea is to re-examine the Bohr model first by taking into account the magnetic field created in the atom due to the electron circulation. This field was fully neglected in the original Bohr's approach. But in effect of the magnetic supplement the electron is moving not in one but in two fields, i.e. the electric and magnetic together. For a combination of such fields the Lorentz force acting on the atomic electron can be easily calculated [6].

We find that the Lorentz force for the hydrogen electron becomes exactly zero. A similar calculation can be done for the classical radiation intensity of the electron particle. This provides us also with a zero result for that intensity. It should be noted that both zero effects are strictly connected with the quantization properties exhibited by the electric and magnetic fields entering the atom.

\section{A Look on the Electron Properties in the Hydrogen Atom}

A specific situation of the Bohr hydrogen atom is that all discrete states of that atom are the bound states, so they have a negative energy. Since the electron energy in the atom obeys the virial theorem [7], viz.

$$
2 E_{\text {kin }}+E_{\text {pot }}=0
$$

where $E_{\text {kin }}$ and $E_{\text {pot }}$ are respectively the kinetic and potential energy parts, the total energy of a quantum state $n$ becomes

$$
E_{\mathrm{tot}}=E_{\mathrm{kin}}+E_{\mathrm{pot}}=-E_{\mathrm{kin}}
$$

which is a negative kinetic electron energy in the $n$ state. The kinetic energy can be written as

$$
E_{\text {kin }}=\left(\frac{1}{2}\right) m v_{n}^{2}=\frac{1}{2} m\left(\frac{e^{2}}{n \hbar}\right)^{2}
$$

since [7]

$$
v_{n}=\frac{e^{2}}{n \hbar}
$$

and $m$ is the electron mass. Evidently the lowest quantum state $n=1$ entering (2) should have the largest velocity

$$
v_{1}=\frac{e^{2}}{\hbar}
$$

because this makes (2) of the lowest value for any $n \geq 1$. On the other hand the velocity $v_{n}$ decreases gradually to zero at infinitely high $n$; see (4).

Classically the circulating electron in the atom is submitted to the well-known attractive electric field of the atomic nucleus having the charge $Z e=e$, the property applied widely by Bohr. This gives the well-known electric field acting on the electron [7]:

$$
\vec{E}_{n}=-\frac{e}{r_{n}^{2}} \frac{\vec{r}_{n}}{r_{n}}=-\frac{e^{5} m^{2}}{n^{4} \hbar^{4}} \frac{\vec{r}_{n}}{r_{n}}
$$


But the circular electron motion along an orbit creates also the magnetic field the size of which is dictated by the speed $v_{n}$ in (4). The quanta of this field - neglected by Bohr - are derived below; see also [8].

First, it can be easily verified that $v_{n}$ is coupled with the orbit radius

$$
r_{n}=\frac{n^{2} \hbar^{2}}{m e^{2}}
$$

and the time period $T_{n}$ necessary for the electron travel along the orbit having the radius $r_{n}$, viz.

$$
T_{n}=\frac{2 \pi n^{3} \hbar^{3}}{m e^{4}}
$$

by the formula

$$
v_{n}=\frac{2 \pi r_{n}}{T_{n}}
$$

In effect the electron motion has its circular frequency

$$
\Omega_{n}=\frac{2 \pi}{T_{n}}
$$

and this quantity is induced by the magnetic field $B_{n}$ coupled with (9) by the formula [6] [8] [9]

$$
\Omega_{n}=\frac{e B_{n}}{m c} .
$$

In result be obtain the size of the magnetic field $B_{n}$ due to the electron orbital motion equal to:

$$
B_{n}=\frac{c m^{2} e^{3}}{n^{3} \hbar^{3}}
$$

Our aim is to examine a classical expression for the particle acceleration in the presence of the electric field $\vec{E}$ and magnetic field $\vec{B}$ represented by [6]:

$$
\dot{\vec{v}}_{n}=\frac{e}{m} \sqrt{1-\frac{v^{2}}{c^{2}}}\left\{\vec{E}_{n}+\frac{1}{c}\left[\vec{v}_{n} \times \vec{B}_{n}\right]-\frac{1}{c^{2}} \vec{v}_{n}\left(\vec{v}_{n} \vec{E}_{n}\right)\right\} .
$$

\section{Calculation of the Acceleration in with the Aid of $\vec{E}_{n}, \vec{B}_{n}$, and $\vec{v}_{n}$}

First we note that the electron velocity vector $\vec{v}_{n}$ is normal to the vector $\vec{E}_{n}$, so the dot product

$$
\vec{v}_{n} \cdot \vec{E}_{n}=0
$$

In this way the last expression on the right-hand side of (12) is equal to zero. The next point, rather evident, is that

$$
v_{n}<c
$$

A maximal $v_{n}$ holds for $n=1$ and this gives approximately the ratio

$$
\frac{v_{1}}{c}=\frac{e^{2}}{c \hbar} \cong \frac{1}{137} .
$$


This provides us with the right-hand side of (12) reduced to

$$
\left(\frac{e}{m}\right)\left\{\vec{E}_{n}+\left(\frac{1}{c}\right)\left[\vec{v}_{n} \times \vec{B}_{n}\right]\right\} .
$$

But the second vector component in (16) contains $\vec{v}_{n}$ which is normal to $\vec{B}_{n}$. The resulted vector product lies in the orbit plane and is normal to the electron track, as it does also the vector $\vec{E}_{n}$. In effect $\vec{E}_{n}$ and the vector combining $\vec{v}_{n}$ and $\vec{B}_{n}$ can add together. We find:

$$
\left(\frac{1}{c}\right)\left[\vec{v}_{n} \times \vec{B}_{n}\right]=\frac{1}{c} \frac{e^{2}}{n \hbar} \frac{c m^{2} e^{3}}{n^{3} \hbar^{3}} \frac{\vec{r}_{n}}{r_{n}}=\frac{e^{5} m^{2}}{n^{4} \hbar^{4}} \frac{\vec{r}_{n}}{r_{n}}
$$

because the sizes of both vectors entering (17) which are normal each to other are constant in time. In the next step let us note that the size of (17) is equal to the size of $\vec{E}_{n}$ in (5).

Since the results obtained in (5) and (17) have opposite signs, the sum in (16) becomes equal to zero. In effect we obtain a vanishing Lorentz force due to (12) acting on the electron for any quantum number $n$.

\section{The Energy Radiated by the Electron in State $n$}

This energy is obtained for the electron particle being in the quantum state $n$ in the form of the integral [6]:

$$
\Delta E_{n}=\frac{2 e^{4}}{3 m^{2} c^{3}} \int_{-\infty}^{+\infty} \frac{\left(\vec{E}_{n}+\frac{1}{c}\left[\vec{v}_{n} \times \vec{B}_{n}\right]\right)^{2}-\frac{1}{c^{2}}\left(\vec{E}_{n} \cdot \vec{v}_{n}\right)^{2}}{1-\frac{v_{n}^{2}}{c^{2}}} d t .
$$

Evidently the expression entering the numerator of the fraction under the integral is equal to zero because of the result in (13) and

$$
\vec{E}_{n}+\left(\frac{1}{c}\right)\left[\vec{v}_{n} \times \vec{B}_{n}\right]=0
$$

entering equally (16) and (18). Therefore no energy is radiated classically by the electron being in state $n$.

\section{Reference of the Field Strengths $E_{n}$ and $B_{n}$ to the Electron Spin}

The spin of the electron particle has been fully neglected in the Bohr atomic model. Nevertheless it can be considered in that model on the basis of the papers [10] [11] [12].

The approach done in references [10] [11] [12] concerned mainly the spin property of electron on the Bohr quantum level $n=1$. Our aim is to extend the spin discussion to the orbits having $n>1$. First, the assumption that the spinning electron particle is circulating with velocity close to the light speed $c$ along a circle having its radius equal to the shortest distance acceptable between two electron particles, viz.

$$
\Delta x_{\min }=r_{c}=\frac{\hbar}{m c} \approx \frac{c}{\Omega_{c}}
$$

remains unchanged. The $\Omega_{c}$ is the angular velocity of the spinning electron. 
The motion chracterized by the radius $r_{c}$ in (20) induces the magnetic field of the strength $B_{c}$ which is coupled with $\Omega_{c}$ by the formula

$$
\Omega_{c}=\frac{e B_{c}}{m c} .
$$

This expression, together with (20), implies the relation

$$
\frac{\hbar}{m c} \approx \frac{m c^{2}}{e B_{c}}
$$

so

$$
B_{c}=\frac{m^{2} c^{3}}{e \hbar} .
$$

Simultaneously a minimal distance between the proton being at rest and the moving electron particle should approach also the distance (20) because the proton contribution to that distance is negligible due to a large proton mass. In effect the absolute value of the electrostatic force between the proton and the spinning electron becomes [11] [12]:

$$
e E_{c}=\frac{e^{2}}{\left(\Delta x_{\min }\right)^{2}}=\frac{e^{2} m^{2} c^{2}}{\hbar^{2}} .
$$

Since the force $e B_{c}$ is normal to $e E_{c}$, the driving electron velocity due to the joint action of $e B_{c}$ and $e E_{c}$ is given by [13]:

$$
v_{c}=c \frac{\left|\vec{E}_{c} \times \vec{B}_{c}\right|}{B_{c}^{2}}=c \frac{E_{c}}{B_{c}}=c \frac{e^{2}}{\hbar c}=\frac{e^{2}}{\hbar} .
$$

Therefore the driving velocity (25) is equal to the orbital Bohr electron velocity existent in the case of $n=1$; see (4a).

But the ratio $\frac{E_{c}}{B_{c}}$ entering $(25)$ is exactly equal to $\frac{E_{1}}{B_{1}}$, so we have

$$
c \frac{E_{c}}{B_{c}}=c \frac{E_{1}}{B_{1}}=v_{1} .
$$

This is a maximal driving velocity along the Bohr orbit. The other orbit velocities like

$$
v_{2}, v_{3}, v_{4}, \ldots<v_{1}
$$

are smaller than $v_{1}$ and can be attained successively by the ratios:

$$
\begin{aligned}
& c \frac{E_{2}}{B_{2}}=c \frac{e^{2}}{2 \hbar c}=\frac{e^{2}}{2 \hbar}=v_{2}, \\
& c \frac{E_{3}}{B_{3}}=c \frac{e^{2}}{3 \hbar c}=v_{3}, \\
& c \frac{E_{4}}{B_{4}}=c \frac{e^{2}}{4 \hbar c}=v_{4},
\end{aligned}
$$

etc. This means that the driving electron velocities along successive orbits $n$ can be provided by the ratios $\frac{E_{n}}{B_{n}}$ characteristic for these orbits, with no reference to the ratio $\frac{E_{c}}{B_{c}}$ excepting for the case of $n=1$.

The parameters entering $E_{c}$ and $B_{c}$ define the electron spin frequency which is the same for all Bohr orbits $n$. Since the time of circulation along the spin orbit having the radius $r_{c}$ is equal to

$$
T_{c} \cong \frac{2 \pi r_{c}}{c}=\frac{2 \pi \hbar}{m c^{2}}=\frac{h}{m c^{2}},
$$


the number of spin circulations in course of the time $T_{n}$ necessary to travel along the $n$th Bohr orbit becomes [see (7)]:

$$
\frac{T_{n}}{T_{c}}=\frac{2 \pi \hbar^{3} n^{3}}{m e^{4}} \frac{m c^{2}}{h}=n^{3} \frac{\hbar^{2} c^{2}}{e^{4}}=\frac{n^{3}}{\alpha^{2}} .
$$

Here $\alpha$ is the well-known atomic constant:

$$
\alpha=\frac{e^{2}}{\hbar c}
$$

The angular frequency of a single spin circulation equal to

$$
\Omega_{c}=\frac{2 \pi}{T_{c}}=\frac{m c^{2}}{\hbar}
$$

is evidently independent of $n$.

\section{Adiabatic Invariants for the Electron Orbital Motion and Spin Motion in the Bohr Atom}

Having the $B_{n}$ for the electron orbital motion and $B_{c}$ for the spin motion [see Equations (11) and (23) respectively] it is easy to calculate the adiabatic invariant in each motion case according to the formula [6]:

$$
I=\frac{3 \pi c p_{n}^{2}}{e B_{n}}
$$

The symbol $p_{n}$ in (32) represents the size of momentum along the electron path which is

$$
p_{n}=m v_{n}=m \frac{e^{2}}{n \hbar}
$$

in the case of circulation along the $n$th Bohr orbit with the velocity $v_{n}$ [see (4)], and instead of $p_{n}$ the expression

$$
p_{c}=m c
$$

approximates the momentum of a spinning electron particle; see the inferences above (20).

The formula (33) substituted to (32) together with $B_{n}$ from (11) gives the invariant equal to

$$
I=I_{n}=\frac{3 \pi c}{e^{4} c m^{2}} n^{3} \hbar^{3} \frac{m^{2} e^{4}}{n^{2} \hbar^{2}}=3 \pi n \hbar,
$$

whereas (34) substituted to (32) instead of $p_{n}$ and $B_{c}$ of (23) instead of $B_{n}$ lead to the invariant formula:

$$
I=\frac{3 \pi c p_{c}^{2}}{e B_{c}}=\frac{3 \pi c m^{2} c^{2}}{m^{2} c^{3}} \hbar=3 \pi \hbar .
$$

Evidently (35) and (36) are much similar: they differ solely by an integer factor $n$ indicating the orbit number present in (35). 


\section{Summary}

The Lorentz force acting on the electron in the Bohr's hydrogen atom, and classical radiation energy of that electron which is assumed to occupy a definite quantum state $n$, are examined. It is found that both the Lorentz force and radiation energy tend to be zero, on condition the magnetic field induced by the electron motion in the atom, neglected in the former Bohr's theory, is taken into account.

The electric and magnetic field strengths ( $E_{n}$ and $B_{n}$ respectively) entering the Lorentz force are useful in deriving the drift velocity of the electron along the orbit $n$. For $n=1$ the drift velocity obtained from $E_{n}$ and $B_{n}$ is equal to that obtained from the ratio of $E_{c}$ and $B_{c}$ characteristic for the spinning electron particle. But for $n>1$ the driving velocity, equal to $v_{n}<v_{1}$, is defined solely by the ratio of $E_{n}$ and $B_{n}$.

A characteristic feature concerns the adiabatic invariants of the orbital and spin motion calculated respectively in Section 6: both invariants differ solely by the integer factor $n$ present in the orbital case.

\section{Conflicts of Interest}

The author declares no competing financial interests.

\section{References}

[1] Bohr, N. (1913) The London, Edinburgh, and Dublin Philosophical Magazine and Journal of Science, 26, 857-875. https://doi.org/10.1080/14786441308635031

[2] Bohr, N. (1914) The London, Edinburgh, and Dublin Philosophical Magazine and Journal of Science, 27, 506-524. https://doi.org/10.1080/14786440308635119

[3] Bohr, N. (1915) Philosophical Magazine, 29, 332.

[4] Bohr, N. (1915) The London, Edinburgh, and Dublin Philosophical Magazine and Journal of Science, 30, 394-415. https://doi.org/10.1080/14786440908635413

[5] Bohr, N. (1922) The Theory of Spectra and the Atomic Constitution. Cambridge University Press, Cambridge, UK.

[6] Landau, L.D. and Lifshitz, E.M. (1948) The Field Theory. 2nd Edition, OGIZ, Moscow. (In Russian)

[7] Sommerfeld, A. (1931) Atombau und Spektrallinien. Vol. 1, 5th Edition, Vieweg, Braunschweig.

[8] Olszewski, S. (2016) Quantum Matter, 5, 641-644. https://doi.org/10.1166/qm.2016.1357

[9] Olszewski, S. (2016) Reviews in Theoretical Science, 4, 336-352. https://doi.org/10.1166/rits.2016.1066

[10] Olszewski, S. (2014) Journal of Modern Physics, 5, 1254.

[11] Olszewski, S. (2014) Journal of Modern Physics, 5, 2022-2029. https://doi.org/10.4236/jmp.2014.518198 
[12] Olszewski, S. (2014) Journal of Modern Physics, 5, 2030-2040. https://doi.org/10.4236/jmp.2014.518199

[13] Matveev, A.N. (1964) Electrodynamics and the Theory of Relativity. Izd. Wyzszaja Szkola, Moscow. (In Russian) 